Arch. Vet. Scienc., 3(1):119-122, 1998

Printed in Brazil

\title{
DESENVOLVIMENTO DE MÉTODOS FÍSICOS PARA O ESTUDO DO COMPORTAMENTO BIOQUÍMICO E FISIOLÓGICO DE ORGANISMOS ANTÁRTICOS. II. POLARÓGRAFO COM ELETRODOS DE IMPLANTE EM TECIDOS.
}

\author{
PEDRO HELIO LUCCHIARI ${ }^{1}$; MATEUS SUGIZAKI ${ }^{2}$; MAURÍCIO BACILA ${ }^{3}$; \\ MARIA IVETTE CARBONI MALUCELLI ${ }^{4}$; \\ METRY BACILA ${ }^{5}$
}

\author{
${ }^{1}$ Departamento de Medicina, Centro de Ciências Biológicas e da Saúde, Pontifícia Universidade Católica do \\ Paraná-PUC-PR, Curitiba. ${ }^{2}$ Faculdade de Ciências, UNESP, Campus de Bauru-SP. ${ }^{3}$ Centro de Ciências Agrárias \\ e Ambientais, Pontifícia Universidade Católica do Paraná - PUC-PR, São José dos Pinhais, PR. ${ }^{4}$ Instituto \\ Butantan, São Paulo-SP. ${ }^{5}$ Departamento de Medicina, Centro de Ciências Biológicas e da Saúde, Pontifícia \\ Universidade Católica do Paraná - PUC-PR, Curitiba e Laboratório de Piscicultura, Curso de Pós-Graduação em \\ Ciências Veterinárias, Universidade Federal do Paraná.
}

\section{Introdução}

O método polarográfico para a medida do oxigênio dissolvido numa solução, na sua forma mais simples, consiste na medida da corrente elétrica entre dois eletrodo, um de metal nobre (geralmente platina) e outro de referência (geralmente prata/cloreto de prata ou calomelano). Movidos pelo interesse no desenvolvimento de diferentes projetos relacionados com o comportamento bioquímico e fisiológico animal, propôs-se a criação de metodologia própria, através de eletrodos de implante, "in vivo", usando o método polarográfico. Nesse particular, foram projetados e desenvolvidos métodos especiais destinados, principalmente, ao estudo dos processos respiratórios teciduais em organismos intactos.

\section{Considerações Gerais}

LUCCHIARI e HOSHINO (1980) desenvolveram um eletrodo (Fig. 1 A) do tipo agulha (platina/prata cloreto de prata) e o respectivo circuito polarográfico e o inseriram esteriotaxicamente na formação reticular mesencefálica de ratos. O par de eletrodos por eles empregado foi construído utilizando-se fios de platina e prata de $0,3 \mathrm{~mm}$ de diâmetro por $30 \mathrm{~mm}$ de comprimento, cada um, isolados entre si com verniz inerte e fixados paralelamente um ao outro com próteses de acrílico. A ponta livre foi cortada em bisel no comprimento desejado, imediatamente antes de sua inserção. Submeteram o animal a varias manobras, tais como, administração de pentobarbital sódico, ketamina, oxigênio puro, carbogênio, asfixia e observaram os efeitos sobre a atividade neuronal local.

CICOGNA et al. (1986a, 1986b), CICOGNA et al. (1988a, 1988b) e CICOGNA et al. (1989)

Correspondência para: Prof. Pedro Helio Lucchiari, Departamento de Medicina, Centro de Ciências Biológicas e da Saúde, Pontifícia Universidade Católica do Paraná - PUC-PR. estudaram as variações dos níveis da tensão de oxigênio no miocárdio e no seio coronário de cães anestesiados, pela infusão de bloqueadores de cálcio, drogas simpatomiméticas, e pela asfixia, reoxigenação e reperfusão cardíaca, através do implante de eletrodos polarográficos. O eletrodo (Fig. 1B) foi construído a partir de um fio de platina de $0,183 \mathrm{~mm}$ de diâmetro, revestido com verniz isolante e fixado numa peça de plástico de aproximadamente seis milímetros de diâmetro e inserido perpendicularmente na superfície do miocárdio. As medidas no seio coronário foram feitas com eletrodo de platina tipo catéter. O eletrodo de referência foi o de calomelano, posto em contato com o sangue circulante através de ponte salina. O circuito polarográfico utilizado foi o mesmo de LUCCHIARI e HOSHINO (1980).

MEDINA et al. (1967) trabalhando com o método polarográfico estudaram o comportamento fisiológico do músculo cardíaco de sapo sob condições experimentais. Determinaram o consumo de oxigênio do coração isolado pela medida da variação da concentração do oxigênio no líquido de perfusão. Estudaram a ação de drogas com propriedades farmacodinâmicas conhecidas sobre o músculo cardíaco como epinefrina, acetilcolina e atropina.

VERCESI et al. (1992) e VERCESI et al. (1995) estudaram a ação do manitol na isquemia renal no cão. SAKATE et al. (1988) estudaram os níveis do oxigênio no tumor de Walker 256, inoculado na coxa de rato, antes e após a irradiação com Cobalto 60 e compararam os resultados com os do músculo normal. Todas as determinações foram conduzidas com o mesmo sistema de eletrodos de implante utilizado por LUCCHIARI et al. (1984).

Paralelamente ao crescente interesse pela aplicação do eletrodo de platina nas medidas da tensão de oxigênio "in vivo", desenvolveram-se também técnicas na preparação "in vitro", ou viceversa. ESTABROOK (1967) enfatizou o uso do método polarográfico pela sua conveniência, simplicidade e rapidez nas medidas da utilização 
do oxigênio pelas células e tecidos, vinculando o tipo e o projeto do eletrodo para oxigênio na dependência da habilidade do investigador e das necessidades específicas do sistema sob investigação.

A posição do eletrodo de referência com relação ao eletrodo de platina variou de autor para autor. Pode-se afirmar, em termos gerais, que sempre houve preocupação em aproximar o eletrodo de referência tanto quanto possível do eletrodo de platina. Em muitos casos, uma ponte salina de cloreto de potássio foi utilizada para pôr em contato o eletrodo de referência com a preparação.

Outra contribuição importante dada ao emprego dos eletrodos para medida da concentração de oxigênio, foi a do aprimoramento dos circuitos eletrônicos usados para alimentar os eletrodos e amplificar a corrente polarográfica. Basicamente, a polarização do eletrodo de platina foi sempre feita com uma pilha comum de 1,5 V. A corrente de polarização medida pela queda de potencial num resistor, sendo este variável e o seu valor ajustado à sensibilidade desejada, como foi utilizado e descrito por ESTABOOK (1967). Dependendo da sensibilidade do registrador, um pré-amplificador era ou não intercalado.

Uma das maiores preocupações com relação ao uso do eletrodo de platina é a sua tendência ao "envelhecimento", que é caracterizada por um declínio lento da corrente de polarização por períodos de minutos ou horas, mesmo que a tensão de oxigênio do meio seja mantida constante. Este fenômeno permanece ainda hoje como sendo um verdadeiro mistério, o que torna a feitura de bons eletrodos um processo que envolve cuidados particulares para cada caso, não existindo, portanto, uma solução de caráter universal. Em todos os experimentos, as medidas foram feitas com eletrodos preparados minutos antes de sua inserção, cuidado que minimizou bastante o problema do envelhecimento. Eletrodos não utilizados foram armazenados em frasco âmbar contendo solução de $\mathrm{NaCl}$, que é uma prática recomendada para evitar os problemas do envelhecimento (DAVIES, 1962).

Fig. 1. Representação esquemática dos eletrodos construídos para medida da tensão de oxigênio. A -Intracerbral de rato; B Miocárdio de cão; C - Músculo de peixe e outros órgãos e tecidos de animais de laboratório.

LUCCHIARI e HOSHINO (1980) implantando eletrodos bipolares no cérebro de ratos, evidenciaram o fato de que o método polarográfico permite a detecção das variações relativas da tensão de oxigênio intracerebral e que pode ser empregado com grande eficácia na investigação de problemas biológicos. A análise destes eletrodos revela sua baixa toxicidade, devido à baixa solubilidade do cloreto de prata. A conjugação dos eletrodos permite, por sua vez, a inserção intracerebral com uma só penetração esteriotáxica. A manutenção das pontas do eletrodo em posição fixa e próximas, dentro de um mesmo padrão, permite assegurar a dependência local das medidas obtidas, além de diminuir os problemas de ruídos que advém do distanciamento dos eletrodos.

CICOGNA et al. (1986a, 1986b), CICOGNA et al. (1988a, 1988b) e CICOGNA et al. (1989) implantando eletrodos monopolares, do tipo agulha, em miocárdio de cão, colocaram em discussão as possíveis causas de erro no uso dos eletrodos de implante: a) coeficiente de difusão do oxigênio nos tecidos; b) consumo de oxigênio pelos eletrodos; c) deposição de proteínas ou reações químicas na 
superfície do eletrodo; d) deslocamento da ponta do eletrodo ou movimentos de líquido na sua extremidade; e) dimensões do eletrodo; f) consumo de oxigênio pelos eletrodos.

A despeito do grande número de informações nas trocas de oxigênio e de gás carbônico do sangue de peixes, a tensão dos gases era medida apenas indiretamente. Afim de poder melhor estudar o oxigênio disponível em tecidos de peixes, à semelhança de inúmeros trabalhos realizados em animais de laboratório, LUCCHIARI et al. (1984) desenvolveram um eletrodo de implante para determinações polarográficas do oxigênio, simultaneamente acoplado com um sistema de medida de $\mathrm{pH}$, salinidade, temperatura, e concentração de oxigênio da água numa câmara de teste. Os testes foram feitos com carpa, Cyprinus carpio, e o eletrodo de implante (Fig. 1C) foi construído a partir de dois fios de $0,12 \mathrm{~mm}$ de diâmetro, sendo um de prata (referência) e outro de platina (medida). Ambos foram revestidos com uma fina camada isolante de resina Epoxi e fixados perpendicularmente a uma delgada base de plástico de 4 por $7 \mathrm{~mm}$ e a uma distância de 3 a $6 \mathrm{~mm}$ um do outro. Às extremidades dos fios voltadas para a base, foram soldados dois fios condutores flexíveis, de pequeno calibre, e a solda recoberta com uma camada isolante de resina epoxi. Imediatamente antes da inserção, as pontas dos eletrodos foram cortadas no comprimento desejado, expondo assim as pontas dos fios de prata e platina. Os eletrodos foram inseridos laterodorsalmente, próximos à coluna vertebral, através de um corte na pele de aproximadamente $2 \mathrm{~cm}$. O músculo foi separado da pele logo abaixo do corte e logo após o implante, fechado com dois pontos cirúrgicos. Para poder manter e conter o peixe em condições normais, LUCCHIARI et al. (1984), projetaram e construíram um tanque em acrílico (Fig. 2), com capacidade de 2 litros, fixado no interior de um banho de água circulante o qual pode manter constante a temperatura. $\mathrm{O}$ gás borbulhado na câmara teste passava primeiro por uma serpentina de vidro fixada na câmara banho de água circulante, permitido assim que o gás entrasse em equilíbrio térmico com a água. A tampa da câmara era removível e possuía orifícios por onde podiam passar os sensores e cabos condutores.

Fig. 2. Representação esquemática da câmara experimental para o estudo da respiração de peixes com eletrodo de oxigênio implantado. CV = Tampa; GD = Difusor de gás; EC = Câmara experimental; OEI = Eletrodo de oxigênio (LUCCHIARI, et al., 1983).

O trabalho de LUCCHIARI et al. (1984) foi de fundamental importância e representou um avanço no estudo da respiração de peixes in vivo, dando origem ao desenvolvimento de outros trabalhos, principalmente relacionados com o comportamento fisiológico de peixes antárticos como em FANTA et al. (1990), LUCCHIARI et al. (1990), FANTA et al. (1989), FANTA et al. (1990) e BASTOS-RAMOS et al. (1998).

Importante passo para o estudo do metabolismo respiratório de peixes foi dado por LUCCHIARI et al. (1983), estabelecendo um método de implante de eletrodos no músculo de peixe intacto. Nesse importante campo da ciência, muitos achados científicos foram obtidos por uma multiplicidade de técnicas, sob as mais variadas condições. Em alguns casos até mesmo com o animal fora d'água e anestesiado. Em nossos experimentos os animais foram submetidos a uma situação tão natural quanto 
possível. Assim, a câmara de teste usada nesse experimento é pouco estressante para o animal, o qual podia respirar normalmente sem produção de muco, após o período de adaptação. A câmara de teste possibilitou que simultaneamente com as medidas de níveis de oxigênio muscular, fosse possível também monitorar o oxigênio, a temperatura e a salinidade da água da câmara. $\mathrm{O}$ método proposto pode ser usado na obtenção de uma série de informações, podendo também ser adaptado a outras espécies de peixes, ampliando o campo da fisiologia respiratória desses animais, como foi o caso dos peixes antárticos (FANTA, 1989; LUCCHIARI, 1989a, b).

VERCESI et al. (1992 e 1995) trabalhando com os mesmos eletrodos de LUCCHIARI et al. (1984), desenvolveram estudo polarográfico do oxigênio e da isquemia renal no cão e os efeitos da ação do manitol. SAKATE et al. (1987) compararam os níveis de oxigênio no tumor de Walker 256 em rato submetido à radioterapia, com $2000 \mathrm{rad}$, e compararam os resultados com os do músculo normal.

$\mathrm{O}$ nível de oxigênio medido com um eletrodo polarográfico é o resultado da soma de dois níveis. $\mathrm{O}$ primeiro é o consumo ativo do oxigênio pela estrutura biológica em estudo e o segundo é o consumo do próprio eletrodo. Considerando que o consumo de oxigênio do tecido cerebral está em torno de $0,55 \mathrm{x}$ $10,3 \mathrm{ml} / \mathrm{g} / \mathrm{s}$ e que, segundo CATER et al. (1950), o consumo de oxigênio pelo eletrodo de é 1,23 x 10-8 $\mathrm{ml} / \mathrm{s}$, concluímos que $1 \mathrm{~g}$ de tecido cerebral consome, durante as medidas e no mesmo intervalo de tempo, cerca de 50000 vezes mais que o eletrodo de oxigênio. Nessas condições, as medidas de concentração de oxigênio efetuadas com o eletrodo de oxigênio representam efetivamente a quantidade de oxigênio disponível para as células e tecidos.

\section{REFERÊNCIAS}

BASTOS-RAMOS, W.P.; GONÇALVES， N.M.F.M.; BACILA, M. Cholinergic bradycardia temperature induced in Antarctic fishes Notothenia neglecta and Chaenocephalus aceratus. Proc. NIPR Symp. Polar Biol. 11:51-61, 1998.

CICOGNA, A.C.; SPADARO, J.; TUCCI, P.J.F.; CURI, P.R.; LUCCHIARI, P.H. Does the oxygen tension of coronary sinus blood reflect myocardial oxygen tension? Brazilian J. Med Biol. Res. 22:645-648, 1989.

CICOGNA, A.C.; SPADARO, J.; TUCCI, P.J.F.; BREGAGNOLLO, E.A.; RODRIGUES, M.A.M.; CURI, P.R.; LUCCHIARI, P.H. Changes in myocardial and coronary sinus blood oxygen tension induced by asphyxia and reoxygenation. Brazilian $J$. Med. Biol. Res. 21:585-590, 1988a.

CICOGNA, A.C.; SPADARO, J.; TUCCI, P.J.F.; BREGAGNOLLO; LUCCHIARI, P.H. Is coronary sinus blood oxygen tension behavior determined by myocardial oxygen tension variation during cardiac reperfusion? Japanese Heart Journal. 29(2):207-213, 1988 b.
CICOGNA, A.C.; SPADARO, J.; TUCCI, P.J.F.; CURI, P.R.; LUCCHIARI, P.H. Comportamento do oxigênio intramiocárdico durante variações da relação ofertaconsumo de oxigênio pelo coração. II. Efeito de drogas simpatomiméticas. Arq. Bras. Cardiol. 47(2)77-81, 1986a.

CICOGNA, A.C.; SPADARO, J.; TUCCI, P.J.F.; RODRIGUES, M.A.M.; CURI, P.R.; LUCCHIARI, P.H. Comportamento do oxigênio intramiocárdico durante variações da relação oferta-consumo de oxigênio. I. Efeito dos bloqueadores de cálcio. Arq. Bras. Cardiol. 46(6):395-400, 1986 b.

ESTABROOK, R.W. Mitochondrial respiratory control and the polarographic measurement of ADP:O Ratios. In: Methods in enzymology, ESTABROOK, R.W. and PULLMAN, M (Eds.), vol. 10, Academic Press, N. York, 1967, pp. $41-47$.

FANTA, E.; LUCCHIARI,P.H.; BACILA, M. The effect of the enviromental oxygen and carbon dioxide levels on the oxygenation and the behavior of Antarctic fish. Comp. Biochem. Physiol. 93A(4):819-31, 1989.

FANTA, E.; LUCCHIARI, P.H.; BACILA,M. Circadian rhythm of oxygen consumption and oxygen levels in the muscle of Notothenia neglecta (Pisces, Teleostei) Comp. Biochem. Physiol. 96C(1):151-155, 1990.

FANTA, E.; LUCCHIARI, P.H.; BACILA, M. The effect of temperature increase on the behavior of Antarctic fish. Proc.NIPR. Symp. Polar Biol. 2:123-130, 1989.

LUCCHIARI, P.H.; FEOFILOFF, E.F.; BACILA,M. A techinique for the determination of the available oxygen in living carp (Cyprinus carpio) muscle. Comp. Biochem. Physiol. 78A(4):675-679, 1984.

LUCCHIARI, P.H.; FANTA, E.; BACILA, M. The effect of temperature on the muscle oxygen levels in Antarctic fish.. Proc.NIPR. Symp. Polar Biol. 2:117-122, 1989a.

LUCCHIARI,P.H.; HOSHINO K.; LUCCHIARI, P.H.; FANTA, E.; BACILA, M. The effect of temperature on the muscle oxygen levels in Antarctic fish.. Proc.NIPR. Symp. Polar Biol. 2:117-122, 1989b.

LUCCHIARI, P.H.; HOSHINO K. Inovação de circuito polarográfico para a determinação de variações dos níveis de oxigênio em meios biológicos. Rev. Ciênc. Bioemed. 1:51-62, 1980.

MEDINA, H.; VOSS, D.O.; BACILA, M. The deternination of oxigen uptake from perfusion liquids by isolated whole heart. An. da Acad. Bras. Ciênc. 39(1):175-177, 1967.

SAKATE, M.; VERC ESI, L.; HENRY, M.A.C.A; LUCCHIARI, P.H. Polarography of Walker tumor submitted to radiotherapy. Tumori. 73:555-558, 1988.

SUGIZAKI, M.; LUCCHIARI, P.H.; MALUCELLI, M.I.C.; BACILA, M. Respiration and oxidative phosphorylation of mitochondria from tissues and organs of Antarctic fish. Proc. NIPR. Symp. Polar Biol. 10:145-152, 1997.

VERCESI, L.A.P.; HENRY, M.A.C.A.; GOLDBERG, J.; LUCCHIARI, P.H. Estudo polarográfico de oxigênio renal no cão: ação do manitol. Acta Cir. Bras. 6(4):147-51, 1995.

VERCESI, L.A.P.; HENRY, M.A.C.A.; LUCCHIARI, P.H.; RODRIGUES, M.A.M.; GOLDBERG，J. Estudo polarográfico de isquemia renal no cão: ação do manitol. Acta Cir. Bras. 7(4):138-41, 1992. 